\title{
Predictors of Postthrombolysis Outcome and Symptomatic Postthrombolysis Hemorrhage following Intravenous Thrombolysis with Alteplase for Acute Ischemic Stroke
}

\author{
Suman Das ${ }^{1}$ Gourango Prosad Mondal ${ }^{1}$ Ramesh Bhattacharya ${ }^{1}$ Kartick Chandra Ghosh ${ }^{1}$ \\ Sarbajit Das ${ }^{1}$ Hema Krishna Pattem ${ }^{1}$ Shabir Ahmed Paul ${ }^{1} \quad$ Chandrakanta Patra $^{1}$
}

\author{
${ }^{1}$ Department of Neurology, Calcutta National Medical College, \\ Kolkata, India
}

J Neurosci Rural Pract 2020;11:315-324

\begin{abstract}
Address for correspondence Suman Das, MD, Department of Neurology, Calcutta National Medical College, 24, Gorachand Road, Kolkata 700014, India (e-mail: dr.sumands@gmail.com).
\end{abstract}

Keywords

- thrombolysis

- intracranial hemorrhage

- alteplase

- modified Rankin scale

- fibrinogen
Background Thrombolysis improves the outcome in acute ischemic stroke (AIS), albeit with an increased risk of symptomatic intracranial hemorrhage (sICH). Biomarkers to find patients at risk of sICH, and guide treatment and prognosis would be valuable.

Methods Consecutive patients of AIS thrombolysed between February 2017 and September 2019 at Calcutta National Medical College were studied prospectively for sICH and outcome at 6-month follow-up. We identified the independent risk factors for unfavorable outcomes, mortality, and sICH using multivariate analysis. Prethrombolysis and 24-hour postthrombolysis fibrinogen levels were estimated to evaluate its biomarker role.

Results Out of 180 AIS patients admitted during the study period, 60 patients were thrombolysed. Door to needle time was $<3$ hours among 24 patients and 3 to 4.5 hours among 36 patients. Favorable outcomes occurred among $76.67 \%$ and sICH occurred among $13.33 \%$ patients. Upper tertile of National Institute of Health Stroke Scale (NIHSS) had the highest adjusted odds for sICH (17.5 [95\% confidence intervals=1.7-178.44]). Total anterior circulation stroke had the highest adjusted odds for unfavorable outcome (19.11 [3.9-92.6]). Following thrombolysis, the mean (standard deviation) fibrinogen level of 449.27 (32.87) decreased $7 \%$ to postthrombolysis level of 420 (20.5; $p<0.0001)$. Higher tertiles of fibrinogen levels had progressively increasing odds for morbidity and sICH.

Conclusion Congestive heart failure, hypertension, age $\geq 75$ years, diabetes mellitus, stroke (double weight), i.e., CHADS2 score $>2$, low ejection fraction, the occurrence of total anterior circulation stroke and higher mean arterial blood pressure, blood glucose level, NIHSS score, and fibrinogen at admission were the common risk factors significantly predicting postthrombolysis sICH and morbidity. Antiplatelet and anticoagulant therapy, lower ASPECT (Alberta Stroke Program Early CT Score), and higher SEDAN scores also predicted sICH. Fibrinogen levels were significantly higher among those developing sICH and having unfavorable outcome. The performance of thrombolysis within 3 hours or between 3 and 4.5 hours after symptom onset did not affect morbidity, mortality, or the occurrence of sICH.
C2020 Association for Helping Neurosurgical Sick People
License terms

$($ () $\odot \circledast$ 


\section{Introduction}

The stroke in low and middle-income countries (LMICs) has grown to epidemic proportions, currently accounting for twothirds of global stroke occurrence and $85 \%$ of global stroke mortality. ${ }^{1}$ During the past decade, the cumulative incidence of stroke in India ranged from 105 to $152 / 100,000$ persons per year. Around 68 to $83.6 \%$ were ischemic stroke and onethird of the patients lived with moderate to severe poststroke disability. ${ }^{2,3}$ Thrombolysis using r-tPA (recombinant tissue plasminogen activator) recanalizes the occluded vessel and increases the perfusion to the ischemic penumbra which has the capacity to recover. Unfortunately, r-tPA also has a destructive effect on extracellular matrix and endothelial basal lamina compromising the blood-brain barrier and resulting in symptomatic intracranial hemorrhage (sICH). As per the NINDS (National Institute of Neurological Disorders) criteria, sICH complicating thrombolysis has a worldwide incidence rate of 2.2 to $8 \%$, and 4.87 to $7.3 \%$ in China and limited published studies from India have shown marked variation. ${ }^{4-6}$ Higher rates of postthrombolysis sICH occur among Asians due to racial differences in blood coagulation-fibrinolysis factors, which could affect the cost-benefit ratio of r-tPA therapy in Asian LMICs. ${ }^{1}$ So, it is worthwhile to investigate the predictive ability of the potential variables for sICH in the Indian population. None of these variables should contraindicate thrombolysis but should alert the physician about the possible consequences. The investigations for the biomarkers have been directed to single proteins selected on the basis of their relationship to the pathophysiology of ischemic stroke. These include biomarkers of neuronal and endothelial damage, inflammation, and coagulation/thrombosis. The heterogeneous nature of ischemic strokes hindered the successful translation of such proteins to biomarkers useful in clinical practice. Biomarkers are classified on the basis of their pathophysiological actions in ischemic stroke. Markers of neuronal injury include S100 calcium-binding protein B, neuron-specific enolase, myelin basic protein, and glial fibrillary acidic protein. Markers involved in inflammation are C-reactive protein, tumor necrosis factor- $\alpha$, interleukin- 6 , intercellular adhesion molecule 1 , vascular cell adhesion protein 1 , matrix metalloproteinases, and antibodies against $N$-methyld-aspartate receptor. Fibrinogen, von-Willebrand factor, and D-Dimer are the biomarkers of acute thrombosis. ${ }^{7}$

We primarily aimed to establish the independent risk factors for intracranial hemorrhage and poor outcomes on the modified Rankin scale (mRS). We secondarily aimed to investigate the role of serum fibrinogen as a biomarker for ischemic stroke for its association with multiple steps in the pathogenesis of ischemic stroke, like endothelial damage, inflammation, and thrombosis.

\section{Methods}

\section{Study Setting}

The study was conducted at the Neurology Department of Calcutta National Medical College, following approval by the Institutional Ethics Committee.
- Study design-Hospital-based prospective interventional study.

- Study duration-February 2017 to September 2019.

- Inclusion criteria-Consecutive acute ischemic stroke (AIS) patients presenting within 4.5 hours and receiving thrombolysis.

We obtained the written informed consent from the relatives of the patients included in this study. After admission, we recorded a quick history and systemic examination was performed. From the medical history prior to the stroke, we calculated the CHADS2 scores for all patients (CHADS2, congestive heart failure, hypertension, age $\geq 75$ years, diabetes mellitus, stroke [double weight],transient ischemic attack [TIA], or thromboembolism). We analyzed the following variables: age, gender, domestic arrangements (living alone or with family members), ongoing medications, histories of vascular risk factors including hypertension, diabetes mellitus, heart diseases (ischemic heart disease, low ejection fraction [EF], and atrial fibrillation [AF], either as a history of AF or AF diagnosed during the index admission by electrocardiography), TIA, current or former smoking, and hypercholesterolemia. At admission, the stroke severity was ascertained by a neurologist experienced in NIHSS evaluation and was classified as mild (0-4), moderate (5-15), or severe (16-42). According to the Bamford criteria, ${ }^{8}$ strokes were classified in total anterior circulation stroke (TACS), partial anterior circulation stroke, posterior circulation stroke, and lacunar stroke. Complete hemograms, coagulation profiles, liver, and renal function tests were recorded in the registry at admission and again 24 to 48 hours later. The cardiological profile (electrocardiogram and transthoracic echocardiography) and investigations for vasculitis (antinuclear and antiphospholipid antibodies) were done. The SEDAN and ASPECTS (Alberta Stroke Program Early CT Score) scores were calculated for all patients. The SEDAN score, (0-6 points), which is based on blood sugar, early infarct sign, dense artery sign, age ( $>75$ years), and NIHSS score $(>10)$, had been suggested in European Cooperative Acute Stroke Study to predict the risk of sICH after intravenous thrombolysis. ${ }^{9}$ The patient's vital parameters were monitored using multiparameter monitors. The entire drug amount $(0.9 \mathrm{mg} / \mathrm{kg}$ of alteplase [Actilyse by Boehringer Ingelheim]) was dissolved in the diluent provided by the manufacturer to attain a concentration of $1 \mathrm{mg} / \mathrm{mL}$. Ten percent of the total dose was administered as a bolus and rest as an infusion over 60 minutes, using a syringe pump. Acute stroke management and secondary prevention in these patients followed current European Stroke Organization guidelines. ${ }^{10}$ Repeat brain CT was done 36 hours after admission. Postthrombolysis ICH was defined as any hemorrhage in the brain within 36 hours after thrombolysis which was detected on imaging. ICH was classified as sICH and asymptomatic ICH. sICH was defined as a CT documented hemorrhage associated with clinical deterioration in the form of a decline in NIHSS score of $\geq 4$ points or death. Following discharge, the patients were followed up monthly for 6 months through neurological examination and review of records. The outcome of patients with $\mathrm{sICH}$ and patients without $\mathrm{sICH}$ 
was evaluated by measuring intrahospital mortality and mRS at 6 months and classified as favorable (score $0-1$ ) or unfavorable (score 2-6).

\section{Statistical Analysis}

We entered the data on SPSS 20.0 software. The baseline characteristics were compared in two groups, using student $t$-test for continuous variables and Chi-square test for categorical variables. The risk factors for intracranial hemorrhage and poor outcomes were evaluated using univariate analysis followed by multiple logistic regressions with variables having two-tailed $p$-value $<0.05$, to establish the independent risk factors. The total fibrinogen concentration was examined by tertiles of increasing levels to evaluate for possible threshold effects. The first tertile served as the reference value.

Exclusion criteria-patients who were not thrombolysed and those with inadequate follow-up data were excluded.

\section{Results}

A total of 180 patients were admitted with ischemic stroke during the study period within 24 hours of symptom onset. Eighty patients arrived at the hospital within 4.5 hours. Out of them, six patients had NIHSS score $>25$, six patients had stroke involving more than one-third cerebral hemisphere, two had recent surgery, three had rapidly improving symptoms, two had blood pressure greater than 185/110, one had a myocardial infarction within less than 3 months, hence they were not thrombolysed. The remaining 60 patients were thrombolysed with injection alteplase. The mean time (standard deviation or SD) of the presentation of patients who were thrombolysed was 3.7 (0.8) hours and 7.4 (2.8) hours for the remaining patients.

Among those 60 patients thrombolysed, 24 patients had a mild stroke, 20 had a moderate stroke, and 16 had a severe stroke. A total of 14 (23.33\%) out of 60 thrombolysed patients had mRS score $\geq 2$. Eight $(13.33 \%)$ out of the 60 thrombolysed patients developed sICH, among whom three patients expired. Among these, six patients bled within the region of the infarct, and two patients bled in a region away from the infarct. One patient had an asymptomatic minor hemorrhagic transformation of the infarct. At admission, all the cases of AF were already diagnosed and were on anticoagulants (warfarin) according to the current guidelines. Some patients with low EF were also on warfarin. Patients with a previous history of TIA and ischemic heart disease were on aspirin therapy. No patient was on dual antiplatelet therapy. Patients who had postthrombolysis hemorrhage were treated with vitamin K1 injection, tranexamic acid, and fresh frozen plasma.

In - Table 1, univariate analysis identified higher age, previous TIA, AF, low EF, CHADS2 score $>2$, occurrence of TACS, and nonlacunar type of stroke to be significant predictors of sICH. Among the continuous variables, lower tertile of ASPECT score, and higher tertiles of MABP (mean arterial blood pressure), CBG (capillary blood glucose), fibrinogen level, SEDAN, and the mean NIHSS scores at admission were significantly attributing to $\mathrm{SICH}$. Multivariate analysis identified CHADS2 score >2 (adjusted odds ratio [AOR] $=14.0$ [95\% confidence interval [CI] $=1.59-120.27], p=0.004)$, occurrence of TACS (AOR=6.1 [95\% CI=1.3-32.45], $p=0.04), \mathrm{AF}(\mathrm{AOR}=10.2$ [95\% CI=1.82-60.46], $p=0.028)$, low EF (AOR=16.22 [95\% $\mathrm{Cl}=2.89-90.87], p=0.0056$ ), antiplatelet therapy (AOR=6.19 [95\% Cl=1.84-30.8], $p=0.029$ ), anticoagulant therapy (AOR=11.07 [95\% CI=3.02-66.6], $p=0.009$ ), upper tertiles of NIHSS score (AOR=16.5 [95\% Cl=1.9-170.84], $p=0.02$ ), fibrinogen level (AOR=12.66 [95\% $\mathrm{Cl}=2.1-114.79$ ], $p=0.025)$, MABP $(\mathrm{AOR}=10.8[95 \% \mathrm{Cl}=3.14-110.55], p=0.04), \mathrm{CBG}(\mathrm{AOR}=11.45$ [95\% Cl=2.19-96.45], $p=0.03$ ), SEDAN score (AOR=9.25 [95\% $\mathrm{Cl}=2.37-51.87], p=0.03)$, and lower tertile of ASPECT score (AOR=0.05 [95\% CI=0.006-0.40], $p=0.01$ ) as independent predictors of sICH. Five out of eight patients with $\mathrm{SICH}$, and 31 out of 52 patients without sICH were thrombolysed between 3 and 4.5 hours of symptom onset $(p=1.0)$. Thus, the performance of thrombolysis within 3 hours of symptom onset or between 3 and 4.5 hours did not affect the chance of sICH. sICH was associated with significantly higher chance of unfavorable outcome (odds ratio=16.5 [2.81-96.75]) and mortality (odds ratio=30.6 [2.66-351.9]).

The univariate analysis in - Table 2 established previous TIA, diabetes, low EF, AF, CHADS2 score $\geq 2$, occurrence of TACS, upper tertiles for age, MABP, CBG, NIHSS, and fibrinogen level as predictors of $\mathrm{mRS}$ score $\geq 2$. Patients with lacunar stroke had a significantly lower incidence of $\mathrm{mRS}$ score $\geq 2(p=0.0009)$. Multivariate analysis established previous TIA (AOR= 6.0 [95\% $\mathrm{Cl}=2.14-19.82], p=0.03$ ), low $\mathrm{EF}$ (AOR=12.4 [95\% Cl=2.04-59.9], $p=0.009)$, CHADS2 score $\geq 2$ (AOR=10.16 [95\% Cl=3.03-37.42], $p=0.0014$ ), occurrence of TACS (AOR=18.04 [95\% Cl=4.02-90.36], $p=0.0002$ ), and upper tertiles of age (AOR=7.4 [95\% CI=2.65-49.44], $p=0.03$ ), MABP at admission (AOR=7.48 [95\% Cl=2.14-38.99], $p=0.04$ ), CBG at admission $(\mathrm{AOR}=6.1$ [95\% Cl=2.69-29.17], $p=0.04)$, NIHSS score at admission ( $\mathrm{AOR}=8.0[95 \% \mathrm{Cl}=1779-47.74)] p=0.03$ ), and fibrinogen level (AOR= 8.2 [95\% $\mathrm{Cl}=1.39-47.88], p=0.03$ ) to be independent predictors of unfavorable outcome. We performed thrombolysis within the 3 and 4.5 hours window in nine patients with $\mathrm{mRS}>2$, and in 21 patients with $\mathrm{mRS}$ $<2(p=0.21)$. Hence, the exact door to needle time for conducting thrombolysis within the window of 4.5 hours did not affect the outcome.

The univariate analysis in - Table 3 identified CHADS2 score $>2$, upper tertiles for age, MABP, CBG, NIHSS, and fibrinogen level as the predictors of mortality. Multivariate analysis confirmed CHADS2 score $>2$ (AOR=infinity, $p=0.042$ ), and upper tertiles of MABP (AOR=infinity, $p=0.045$ ), CBG (AOR=22.2 [95\% Cl=2.4-242.73], $p=0.03$ ), NIHSS (AOR=infinity, $p=0.03$ ), and fibrinogen level (AOR=infinity, $p=0.045$ ) to be independent predictors of mortality. Among the 36 patients thrombolysed within the 3 and 4.5 hours window, two patients expired and 34 patients survived $(p=1)$. Hence, the exact time of conducting thrombolysis within the window of 4.5 hours did not affect mortality. 
Table 1 Characteristics of patients who developed postthrombolysis hemorrhage vs those without postthrombolysis hemorrhage

\begin{tabular}{|c|c|c|c|c|}
\hline Characteristics & $\begin{array}{l}\text { Patients with symptomatic } \\
\text { postthrombolysis } \\
\text { hemorrhage } \\
(N=8)\end{array}$ & $\begin{array}{l}\text { Patients without } \\
\text { symptomatic } \\
\text { postthrombolysis } \\
\text { hemorrhage } \\
(N=52)\end{array}$ & OR $(95 \% \mathrm{Cl})$ & $p$-Value \\
\hline Female sex & 3 & 23 & $0.49(0.1-2.3)$ & 0.45 \\
\hline BMI $\left(\mathrm{kg} / \mathrm{m}^{2}\right)$ & $24.8(0.4)$ & $25.1(0.8)$ & & 0.30 \\
\hline Bottom tertile (ref.) & $24.4-24.67, N=2$ & $24.3-25.83, N=24$ & & \\
\hline Middle tertile & $24.68-24.95, N=2$ & $25.84-23.37, N=17$ & $1.4(0.18-11.03)$ & 1.0 \\
\hline Upper tertile & $24.96-65.6, N=4$ & $25.38-25.91, N=11$ & $4.3(0.69-27.51)$ & 0.16 \\
\hline Age & $59.9(5.5)$ & $56.1(4.5)$ & & 0.03 \\
\hline Bottom tertile (ref.) & $54-57.8, N=1$ & $51.6-54.6, N=20$ & & \\
\hline Middle tertile & $57.9-61.7, N=1$ & $54.7-57.7, N=18$ & $1.11(0.06-19.09)$ & 1.0 \\
\hline Upper tertile & $61.8-65.6, N=6$ & $57.8-60.8, N=14$ & $8.57(0.92-79.27)$ & 0.04 \\
\hline \multicolumn{5}{|l|}{ Vascular risk factors } \\
\hline Previous TIA & 4 & 5 & $9.4(1.77-49.66)$ & 0.01 \\
\hline Current smoking & 2 & 13 & $1(0.17-5.57)$ & 1 \\
\hline Ischemic heart disease & 2 & 6 & $2.55(0.41-15.65)$ & 0.58 \\
\hline $\mathrm{AF}$ & 3 & 3 & $9.8(1.54-62.07)$ & 0.02 \\
\hline Low EF (<35\%) & 4 & 3 & $16.33(2.67-99.90)$ & 0.004 \\
\hline \multicolumn{5}{|l|}{ CHADS2 score } \\
\hline $0-1$ & 1 & 35 & $14.41(1.6-126.72)$ & 0.005 \\
\hline$\geq 2$ & 7 & 17 & & \\
\hline \multicolumn{5}{|l|}{ Bamford classification } \\
\hline TACS & 4 & 7 & $6.4(1.29-31.79)$ & 0.03 \\
\hline PACS & 2 & 11 & $1.24(0.21-7.03)$ & 1.0 \\
\hline PCS & 2 & 12 & $1.11(0.19-6.23)$ & 1.0 \\
\hline LS & 0 & 22 & 0 & 0.04 \\
\hline $\begin{array}{l}\text { Mean (SD) NIHSS score } \\
\text { postthrombolysis }\end{array}$ & $17.5(4.0)$ & $11(4)$ & & $<0.0001$ \\
\hline Bottom tertile (ref.) & $13.5-16.83, N=1$ & $7-9.67, N=35$ & & \\
\hline Middle tertile & $16.84-19.71, N=3$ & $9.68-12.35, N=9$ & $11.6(1.08-125.9)$ & 0.04 \\
\hline Upper tertile & $19.18-21.51, N=4$ & $12.36-15.03, N=8$ & $17.5(1.7-178.44)$ & 0.01 \\
\hline $\begin{array}{l}\text { Patients thrombolysed } \\
\text { between } 3 \text { and } 4.5 \mathrm{~h}\end{array}$ & 5 & 31 & $1.12(0.24-5.25)$ & 1.0 \\
\hline \multicolumn{5}{|l|}{ Home medications } \\
\hline Antiplatelet therapy & 5 & 11 & $6.21(1.28-30.11)$ & 0.02 \\
\hline $\begin{array}{l}\text { Antihypertensive } \\
\text { therapy }\end{array}$ & 4 & 17 & $2.05(0.45-9.24)$ & 0.43 \\
\hline Anticoagulant therapy & 4 & 4 & $12(2.14-67.06)$ & 0.007 \\
\hline Statin therapy & 4 & 14 & $2.71(0.55-12.35)$ & 0.22 \\
\hline Mean (SD) ASPECT & $6.75(1.8)$ & $8.2(1.6)$ & & 0.02 \\
\hline Bottom tertile (ref.) & $4.95-6.15, N=4$ & $6.6-7.67, N=7$ & & \\
\hline Middle tertile & $6.16-7.36, N=3$ & $7.68-8.75, N=9$ & $0.58(0.09-3.5)$ & 0.66 \\
\hline Upper tertile & $7.37-8.57, N=1$ & $8.76-9.83, N=36$ & $0.04(0.004-0.50)$ & 0.007 \\
\hline SEDAN score & $3.875(0.78)$ & $3.45(0.22)$ & & 0.0015 \\
\hline Bottom tertile (ref.) & $2.095-2.948, N=2$ & $3.23-376, N=30$ & & \\
\hline
\end{tabular}


Table 1 (continued)

\begin{tabular}{|c|c|c|c|c|}
\hline Characteristics & $\begin{array}{l}\text { Patients with symptomatic } \\
\text { postthrombolysis hge ( } N \\
=8 \text { ) }\end{array}$ & $\begin{array}{l}\text { Patients without } \\
\text { symptomatic } \\
\text { postthrombolysis hge } \\
(N=52)\end{array}$ & OR (95\%Cl) & $p$-Value \\
\hline Middle tertile & $2.949-3.802, N=1$ & $3.377-3.523, N=13$ & $1.15(0.09-13.87)$ & 1.0 \\
\hline Upper tertile & $3.803-4.656, N=5$ & $3.524-3.670, N=9$ & $8.33(1.37-50.47)$ & 0.02 \\
\hline MABP at admission & $112.8(7.4)$ & $107.0(6.8)$ & & 0.03 \\
\hline Bottom tertile (ref.) & $105.4-109.93, N=1$ & $100.2-104.73, N=28$ & & \\
\hline Middle tertile & 109.94-114.87, $N=3$ & $104.74-109.27, N=14$ & $0.16(0.15-1.75)$ & 0.13 \\
\hline Upper tertile & $114.87-119.80, N=4$ & $109.28-113.81, N=10$ & $11.2(1.14-112.52)$ & 0.03 \\
\hline CBG at admission & $174.1(9.2)$ & $159.7(10.8)$ & & 0.007 \\
\hline Bottom tertile (ref.) & $164.9-171.03, N=1$ & $170.5-177.7, N=25$ & & \\
\hline Middle tertile & $171.04-177.17, N=2$ & $177.8-185.0, N=15$ & $3.33(0.27-39.97)$ & 0.55 \\
\hline Upper tertile & $177.17-183.30, N=5$ & $185.1-192.3, N=12$ & 10.41 (1.09-99.29) & 0.02 \\
\hline Fibrinogen (mg/dL) & $468.5(20.85)$ & $441.4(26.4)$ & & 0.007 \\
\hline Bottom tertile (ref.) & $416-438, N=1$ & $415-429.2, N=21$ & & \\
\hline Middle tertile & $439-461, N=2$ & $429.3-443.3, N=22$ & $1.81(0.15-21.61)$ & 1.0 \\
\hline Upper tertile & $462-482, N=5$ & $443.3-457.4, N=9$ & $11.66(1.8-117.59)$ & 0.02 \\
\hline \multicolumn{5}{|l|}{ Outcome } \\
\hline mRS score $>2$ & 6 & 8 & $16.5(2.81-96.75)$ & 0.0012 \\
\hline Mortality & 3 & 1 & $30.6(2.66-351.9)$ & 0.006 \\
\hline
\end{tabular}

Abbreviations: AF, atrial fibrillation; ASPECT, Alberta Stroke Program Early CT score; BMI, body mass index; CBG, capillary blood glucose; CHADS2, congestive heart failure, hypertension, age $\geq 75$ years, diabetes mellitus, stroke [double weight]; Cl, confidence interval; EF, ejection fraction; ENI, early neurological improvement; hge, hemorrhage; LS, lacunar stroke; MABP, mean arterial blood pressure; mRS, modified Rankin scale; NIHSS, National Institute of Health Stroke Scale; OR, odds ratio; PACS, partial anterior circulation stroke; PCS, posterior circulation stroke; ref., reference (The term 'ref.' means that the bottom tertile is considered as the reference for comparing the values of middle and upper tertile); SD, standard deviation; TACS, total anterior circulation stroke; TIA, transient ischemic attack.

Following thrombolysis, the fibrinogen levels decreased significantly from the prethrombolysis level of 449.27 (32.87) to postthrombolysis level of 420 (20.5) ( $p<0.0001)$.

\section{Discussion}

In the developing countries, r-tPA is provided in public sector hospitals for AIS in India, China, Malaysia, Philippines, Thailand, Turkey, Bulgaria, Czech Republic, Estonia, Poland, Slovakia, Ukraine, Argentina, Brazil, and Peru. Prehospital delay (nonrecognition of stroke warning signs and stroke symptoms, cultural and religious barriers, and lack of well-organized ambulance service), financial and infrastructural constraints are the principal barriers of thrombolysis therapy in LMICs. ${ }^{1}$ The proportion of ischemic stroke patients receiving alteplase is $11 \%$ in the ongoing Indo-U.S. National Stroke Registry. ${ }^{11}$

\section{Discussion on Predictors of an Unfavorable Outcome}

In our study, multivariate analysis identified previous TIA, low EF, CHADS2 score $\geq 2$, occurrence of TACS, and upper tertiles of age, MABP at admission, CBG at admission, NIHSS score at admission, and fibrinogen level to be independent predictors of mRS score $\geq 2$. TACS, low EF, followed by CHADS2 score $>2$ had the highest odds for unfavorable outcomes. In
Cetiner's study, pretreatment NIHSS scores, presence of proximal hyperintense middle cerebral artery sign in noncontrast CT brain, cardioembolic stroke, and age $\geq 80$ years were significantly higher in the group with unfavorable functional outcomes. ${ }^{12}$ In the Zhao's study on the factors associated with thrombolysis outcome in AIS patients with AF, heart failure, higher systolic BP, blood glucose, and NIHSS score independently predicted unfavorable outcomes. Thrombolysis independently predisposed to a favorable outcome but did not significantly reduce mortality. ${ }^{13}$ Huang et al. described that patients with no history of diabetes, leukoaraiosis, low blood glucose level, low systolic BP level, and low baseline NIHSS score before thrombolysis had better outcome. ${ }^{14}$ Thus, the findings of the majority of the studies performed worldwide were in unison with our results.

Considering in-hospital mortality alone, multivariate analysis identified CHADS2 score >2, upper tertiles of MABP, CBG, NIHSS, and fibrinogen level as the independent predictor of mortality. In his study, Bateman et al described that the thrombolysed patients had a higher in-hospital mortality rate compared with the nonthrombolysed patients (11.4 vs. $6.8 \%$ ). His multivariate logistic regression showed that older age, Asian/Pacific Islander race, congestive cardiac failure, and $\mathrm{AF} /$ flutter to be independent predictors, whereas, overall ischemic stroke volume, was not a significant 
Table 2 Results of univariate logistic regression analysis model for probability of unfavorable outcome (mRS score $\geq 2$ ) among thrombolysed patients

\begin{tabular}{|c|c|c|c|c|}
\hline Characteristics & mRS score $\geq 2(N=14)$ & mRS score $<2(N=46)$ & OR $(95 \% \mathrm{Cl})$ & $p$-Value \\
\hline Female sex $(N=26)$ & 7 & 19 & $1.4(0.42-4.72)$ & 0.39 \\
\hline BMI & $26.6 \pm 1.6$ & $26.3 \pm 0.67$ & & 0.30 \\
\hline Bottom tertile (ref.) & $25.8-26.33, N=5$ & $25.3-25.9, N=20$ & & \\
\hline Middle tertile & $26.34-26.86, N=4$ & $26-26.6, N=12$ & $1.33(0.29-5.95)$ & 0.4 \\
\hline Upper tertile & $26.87-27.40, N=5$ & $26.7-27.3, N=14$ & $1.42(0.34-5.88)$ & 0.72 \\
\hline Age & $69.6 \pm 2.06$ & $62.11 \pm 10.01$ & & 0.0076 \\
\hline Bottom tertile (ref.) & $66.5-68.5, N=2$ & $52.1-58.77, N=21$ & & \\
\hline Middle tertile & $68.6-70.6, N=4$ & $58.78-65.45, N=15$ & $2.8(0.45-17.31)$ & 0.38 \\
\hline Upper tertile & $70.7-72.7, N=8$ & $65.46-72.13, N=10$ & $8.4(1.49-47.04)$ & 0.01 \\
\hline Hypertension $(N=44)$ & 10 & 34 & $0.88(0.23-3.34)$ & 1.0 \\
\hline Hypercholesterolemia $(N=36)$ & 11 & 25 & $1.9(0.52-7.04)$ & 0.36 \\
\hline Diabetes $(N=27)$ & 10 & 17 & $4.2(1.15-15.72)$ & 0.03 \\
\hline Previous TIA $(N=9)$ & 5 & 4 & $5.83(1.3-26.11)$ & 0.025 \\
\hline Current smoking $(N=15)$ & 4 & 11 & $1.27(0.33-4.8)$ & 0.99 \\
\hline Ischemic heart disease $(N=7)$ & 3 & 4 & $2.86(0.55-14.72)$ & 0.33 \\
\hline $\mathrm{AF}(N=6)$ & 4 & 2 & $8.4(1.3-52.47)$ & 0.02 \\
\hline Low EF $(<35 \%)(N=7)$ & 5 & 2 & $11.66(1.9-69.94)$ & 0.006 \\
\hline CHADS2 score $\geq 2(N=24)$ & 11 & 13 & $9.3(2.22-38.85)$ & 0.0013 \\
\hline $\operatorname{TACS}(N=11)$ & 8 & 3 & $19.11(3.9-92.6)$ & 0.0001 \\
\hline $\operatorname{PACS}(N=13)$ & 4 & 9 & $1.6(0.4-6.46)$ & 0.71 \\
\hline $\operatorname{PCS}(N=14)$ & 2 & 12 & $0.47(0.09-2.4)$ & 0.48 \\
\hline $\mathrm{LS}(N=22)$ & 0 & 22 & 0 & 0.0009 \\
\hline $\begin{array}{l}\text { Patients thrombolysed } \\
\text { between } 3 \text { and } 4.5 \mathrm{~h}(N=36)\end{array}$ & 9 & 27 & $1.26(0.36-4.38)$ & 0.76 \\
\hline NIHSS & $16.82 \pm 4.82$ & $11.4 \pm 3.4$ & & 0.0001 \\
\hline Bottom tertile (ref.) & $12.0-15.21, N=2$ & $8.0-10.2, N=21$ & & \\
\hline Middle tertile & $15.22-18.43, N=4$ & $10.3-12.5, N=15$ & $2.8(0.45-17.31)$ & 0.3 \\
\hline Upper tertile & $18.44-21.65, N=8$ & $12.6-14.8, N=10$ & $8.4(1.49-47.04)$ & 0.01 \\
\hline MABP & $118.20 \pm 5.2$ & $105.85 \pm 9.35$ & & 0.0001 \\
\hline Bottom tertile (ref.) & $112.9-116.43, N=2$ & $96.5-102.7, N=21$ & & \\
\hline Middle tertile & $116.44-119.97, N=5$ & $102.7-108.9, N=13$ & $3.43(0.59-20.01)$ & 0.22 \\
\hline Upper tertile & $119.98-123.51, N=7$ & $109-115.2, N=12$ & $6.41(1.14-35.89)$ & 0.03 \\
\hline CBG & $170.5 \pm 11.0$ & $163.6 \pm 9.7$ & & 0.0276 \\
\hline Bottom tertile (ref.) & $159.5-166.8, N=3$ & $153.9-160.3, N=24$ & & \\
\hline Middle tertile & $166.9-174.2, N=4$ & $160.4-166.8, N=12$ & $2.66(0.51-13.87)$ & 0.3 \\
\hline Upper tertile & $174.2-181.5, N=7$ & $166.9-173.3, N=10$ & $5.6(1.19-26.13)$ & 0.03 \\
\hline Fibrinogen & $460.2 \pm 12.2$ & $452.8 \pm 6.7$ & & 0.0048 \\
\hline Bottom tertile (ref.) & $447.8-455.9, N=2$ & $446.1-450.56, N=18$ & & \\
\hline Middle tertile & $455.9-464.0, N=5$ & $450.57-455.02, N=20$ & $2.5(0.42-14.60)$ & 0.42 \\
\hline Upper tertile & $464.0-472.1, N=7$ & $455.03-459.48, N=8$ & $7.87(1.33-46.62)$ & 0.02 \\
\hline
\end{tabular}

Abbreviations: AF, atrial fibrillation; ASPECT, Alberta Stroke Program Early CT score; BMI, body mass index; CBG, capillary blood glucose; CHADS2, congestive heart failure, hypertension, age $\geq 75$ years, diabetes mellitus, stroke [double weight]; Cl, confidence interval; EF, ejection fraction; LS, lacunar stroke; MABP, mean arterial blood pressure; mRS, modified Rankin scale; NIHSS, National Institute of Health Stroke Scale; OR, odds ratio; PACS, partial anterior circulation stroke; PCS, posterior circulation stroke; ref., reference; SD, standard deviation; TACS, total anterior circulation stroke; TIA, transient ischemic attack. 
Table 3 Results of univariate logistic regression analysis model for probability of mortality among thrombolysed patients

\begin{tabular}{|c|c|c|c|c|}
\hline Characteristics & $\begin{array}{l}\text { Mortality } \\
(N=4)\end{array}$ & $\begin{array}{l}\text { Surviving } \\
(N=56)\end{array}$ & OR $(95 \% \mathrm{Cl})$ & $p$-Value \\
\hline Female $\operatorname{sex}(N=26)$ & 2 & 24 & $1.09(0.14-8.42)$ & 1.0 \\
\hline BMI & $26.45 \pm 0.85$ & $25.80 \pm 0.80$ & & 0.12 \\
\hline Bottom tertile (ref.) & $25.5-26.1, N=1$ & $25.0-25.53, N=20$ & & \\
\hline Middle tertile & $26.1-26.7, N=1$ & $25.54-26.07, N=18$ & $1.11(0.06-19.09)$ & 1.0 \\
\hline Upper tertile & $26.8-27.4, N=2$ & $26.08-26.60, N=18$ & $2.22(0.18-26.62)$ & 0.6 \\
\hline Age & $68.51 \pm 4.01$ & $62.8 \pm 10.6$ & & 0.29 \\
\hline Bottom tertile (ref.) & $64.5-67.17, N=1$ & $52.2-59.2, N=21$ & & \\
\hline Middle tertile & $67.18-69.85, N=2$ & $59.3-66.3, N=23$ & $1.82(0.15-21.63)$ & 1.0 \\
\hline Upper tertile & $69.86-72.53, N=1$ & $66.4-73.4, N=12$ & $1.75(0.1-30.59)$ & 1.0 \\
\hline Hypertension $(N=44)$ & 3 & 41 & $0.36(0.03-4.22)$ & 0.41 \\
\hline Hypercholesterolemia $(N=36)$ & 3 & 33 & $1.18(0.11-12.42)$ & 1 \\
\hline Diabetes $(N=27)$ & 2 & 25 & $0.84(0.1-6.48)$ & 1.0 \\
\hline Previous TIA $(N=9)$ & 2 & 7 & $5.57(0.66-46.38)$ & 0.14 \\
\hline Current smoking $(N=15)$ & 1 & 14 & $0.76(0.07-7.9)$ & 1.0 \\
\hline Ischemic heart disease $(N=7)$ & 2 & 5 & $8.2(0.93-71.7)$ & 0.08 \\
\hline $\mathrm{AF}(N=6)$ & 2 & 4 & $10.5(1.14-95.9)$ & 0.06 \\
\hline Low EF $(<35 \%)(N=7)$ & 2 & 5 & $10.5(1.14-95.9)$ & 0.06 \\
\hline CHADS2 score $\geq 2(N=24)$ & 4 & 20 & Infinity & 0.04 \\
\hline $\operatorname{TACS}(N=11)$ & 2 & 9 & $4.11(0.5-33.27)$ & 0.20 \\
\hline $\operatorname{PACS}(N=13)$ & 1 & 12 & $8.5(0.8-89.74)$ & 0.07 \\
\hline $\operatorname{PCS}(N=14)$ & 1 & 13 & $0.84(0.08-8.89)$ & 1 \\
\hline $\operatorname{LS}(N=22)$ & 0 & 22 & 0 & 0.12 \\
\hline $\begin{array}{l}\text { Patients thrombolysed between } 3 \text { and } 4.5 \mathrm{~h} \\
(N=36)\end{array}$ & 2 & 34 & $0.35(0.04-2.74)$ & 0.56 \\
\hline MABP & $116.2 \pm 7.3$ & $105.6 \pm 9.2$ & & 0.028 \\
\hline Bottom tertile (ref.) & $108.9-114.1, N=0$ & $96.4-102.53, N=29$ & & \\
\hline Middle tertile & $114.2-119.3, N=1$ & $102.54-108.67, N=21$ & Infinity & 0.43 \\
\hline Upper tertile & $119.4-124.6, N=2$ & $108.68-114.81, N=6$ & Infinity & 0.04 \\
\hline CBG & $171.9 \pm 11.0$ & $161.2 \pm 8.2$ & & 0.016 \\
\hline Bottom tertile (ref.) & $160.9-168.2, N=1$ & $153-158.47, N=29$ & & \\
\hline Middle tertile & $168.3-176.5, N=0$ & $158.48-163.95, N=16$ & 0 & 1 \\
\hline Upper tertile & $176.6-183.9, N=2$ & $163.96-169.43, N=11$ & $20(1.4-272.33)$ & 0.03 \\
\hline NIHSS & $16.95 \pm 5.05$ & $12.7 \pm 3.6$ & & 0.02 \\
\hline Bottom tertile (ref.) & $11.9-15.2, N=0$ & $8.1-11.1, N=24$ & & \\
\hline Middle tertile & $15.3-18.6, N=0$ & $11.2-14.2, N=20$ & - & 1 \\
\hline Upper tertile & $18.7-22.4, N=4$ & $14.3-17.3, N=12$ & Infinity & 0.02 \\
\hline Fibrinogen & $472.2 \pm 8.6$ & $450.7 \pm 11.5$ & & 0.0006 \\
\hline Bottom tertile (ref.) & $463.6-469.3, N=0$ & $462.2-469.8, N=23$ & & \\
\hline Middle tertile & $469.4-475.1, N=1$ & $469.9-477.5, N=23$ & Infinity & 1 \\
\hline Upper tertile & $475.2-480.9, N=3$ & $477.6-485.2, N=10$ & Infinity & 0.04 \\
\hline
\end{tabular}

Abbreviations: AF, atrial fibrillation; BMI, body mass index; $\mathrm{CBC}$, capillary blood glucose; $\mathrm{Cl}$, confidence interval; CHADS2, congestive heart failure, hypertension, age $\geq 75$ years, diabetes mellitus, stroke [double weight]; EF, ejection fraction; LS, lacunar stroke; MABP, mean arterial blood pressure; NIHSS, National Institute of Health Stroke Scale; OR, odds ratio; PACS, partial anterior circulation stroke; PCS, posterior circulation stroke; ref., reference; SD, standard deviation; TACS, total anterior circulation stroke; TIA, transient ischemic attack. 
predictor of in-hospital mortality after thrombolysis. ${ }^{15}$ Heuschmann et al reported a 10\% mortality following thrombolysis with advanced age and altered level of consciousness significantly influencing in-hospital deaths. ${ }^{16}$

\section{Discussion on Postthrombolysis Hemorrhage}

Eight (13.33\%) patients had an sICH in our study, which was higher compared with other Indian studies-0 (Padma et al), ${ }^{17} 6.3 \%$ (Sharma and Sharma), ${ }^{18} 1.7 \%$ (Salam et al), ${ }^{195.5 \%}$ (Boddu et $\mathrm{al}$ ), ${ }^{20}$ and other international studies-4.4\% (Bateman et al), ${ }^{15}$ and $7.1 \%$ (Heuschmann et al). ${ }^{16}$ The rate of fatal postthrombolysis hemorrhage was $6.67 \%$ in our study. Whiteley et al meta-analyzed data of 6,756 participants from nine trials of thrombolysis with alteplase versus controls. ${ }^{21}$ Thrombolysis increased parenchymal hemorrhage, occurring in $6.8 \%$ patients allocated to alteplase group versus $1.3 \%$ of controls; and of fatal intracerebral hemorrhage $2.7 \%$ among thrombolysed subjects versus $0.4 \%$ among nonthrombolysed patients. However, the proportional increase in SICH was similar irrespective of age, treatment delay, or baseline NIHSS score, but the absolute excess risk of sICH was elevated with increasing stroke severity ranging from $1.5 \%(0.8-2.6 \%)$ for strokes with NIHSS 0 to 4 to $3.7 \%$ (2.1-6.3\%) for NIHSS 22 or more $(p=0.0101) .{ }^{21}$ In our study, multivariate analysis identified CHADS2 score $>2$, occurrence of TACS, AF, low EF, antiplatelet therapy, anticoagulant therapy, and upper tertiles of NIHSS score, fibrinogen level, MABP, CBG, SEDAN score, and lower tertile of ASPECT score as independent predictors of postthrombolysis hemorrhage. Upper tertile of NIHSS score, low EF, followed by CHADS2 score $>2$, had the highest odds for hemorrhagic conversion. Chenna et al inferred that increased door to needle time, prior diabetes and hypertension, and higher NIHSS score and blood sugar at presentation increased the occurrence of sICH. ${ }^{6}$ The Canadian Alteplase for Stroke Effectiveness Study results showed that the doorto-needle time was an independent predictor of $\mathrm{SICH}$ and there was a nonsignificantly higher trend toward the occurrence of sICH in the 3 to 4.5 hours group compared with 0 to 3 hours group ( 7.8 vs. $3.8 \%, p=0.06$ ). ${ }^{22}$ However, in our study, the occurrence of postthrombolysis hemorrhage was unrelated to the exact time of thrombolysis within the 4.5-hour window period. Cardioembolism, NIHSS on admission $>20$, serum glucose on admission $>9.0 \mathrm{mmol} / \mathrm{L}$, and age $\geqq 70$ years were independent risk factors for sICH in Chinese patients with AIS treated with alteplase, with cardioembolism carrying the highest odds. ${ }^{5}$ Modrego described earlier microbleeds on brain magnetic resonance imaging (MRI), leukoaraiosis, and previous antiplatelet drug use or statin use, uncontrolled hypertension, higher plasma glucose, lower platelet count, surgery within the preceding 3 months, NIHSS $>25$, early signs of infarction on brain CT, ASPECT $\leq 7$, stroke symptoms duration $\geq 4.5$ hours, symptoms of unknown onset time, an apparent diffusion coefficient (ADC) ratio (ADC in the affected area/ADC in the normal contralateral area) $<0.65$, FLAIR (fluid-attenuated inversion recovery) hyperintensity, and focal hyperperfusion in the damaged area after reperfusionon arterial spin labeling in a ratio higher than 1.5 (ipsilateral/contralateral) as independent predictors of sICH. ${ }^{23}$ The increased rate of sICH in our study was possibly because of the fact that among patients who were thrombolysed on the basis of early ischemic changes in brain CT scans, 11 turned out to be TACS on subsequent postthrombolysis imaging and four (36.36\%) patients among them developed hemorrhage. Also, we thrombolysed eight patients on warfarin, among whom four (50\%) developed hemorrhage.

\section{Discussion on Fibrinogen As a Biomarker}

The "early fibrinogen degradation coagulopathy" hypothesis described a biological phenomenon predictive of postthrombolysis hemorrhage related to an early loss of fibrinogen and consequently, leading to the increase of fibrinogen degradation products (FDPs). Studies showed that a decrement in 2 hours postthrombolysis fibrinogen levels to less than $2 \mathrm{~g} / \mathrm{L}$ increased the odds of an early sICH by 12.82 times. $^{24}$ Our study also demonstrated that the serum fibrinogen levels fell significantly after thrombolysis, the mean fibrinogen decreasing by $7 \%(p<0.0001)$ and we recorded an sICH rate of 13.33\%. The Alteplase-Tenecteplase Trial Evaluation for Stroke Thrombolysis study also demonstrated that alteplase was associated with prolongation of prothrombin time, reduction of plasminogen and fibrinogen levels, and elevation of FDP and D-dimer levels. ${ }^{25}$ In their study on whether prethrombolytic plasmatic markers of coagulation and fibrinolysis are correlated to recanalization and functional outcome, Martí-Fàbregas found no relation between fibrinogen level and functional outcome. ${ }^{26}$ However, our study demonstrated that the odds of poor outcome and sICH increased progressively with increasing levels of fibrinogen. AIS predisposes to mitochondrial dysfunction and increased nitric oxide (NO) production which dilates blood vessels, thereby maintaining perfusion. NO stimulates free radical production and generates peroxynitrite, which irreversibly nitrates proteins. During AIS, fibrinogen is upregulated as an acutephase reactant. AIS also increases the permeability of the blood-brain barrier, thereby, allowing the extravasation of plasma proteins into the brain parenchyma. This potentiates fibrinogen nitrotyrosination. Initially, nitro-fibrinogen retards clot formation, but later it produces harmful fibrinolysis-resistant clots and induces brain parenchymal damage. ${ }^{27}$ Immunofluorescence analysis of the affected human brain areas also showed that both fibrinogen and nitrotyrosinated fibrinogen spread into the brain parenchyma after AIS. Measurement of caspase- 3 enzyme activity in the human neuroblastoma cell line assayed the toxicity of fibrinogen and nitrotyrosinated fibrinogen. Nitrotyrosinated fibrinogen induced higher activation of caspase 3 , which is integral in the cellular apoptosis process. Cell survival assays also demonstrated a greater neurotoxic effect of nitrotyrosinated fibrinogen at all tested concentrations. These studies suggest that fibrinogen, and especially nitrotyrosinated fibrinogen, plays a prominent role in the progression of brain tissue damage in the penumbral region, thereby, determining the final extent of the ischemic insult. ${ }^{28,29}$ Hence, higher elevation in the fibrinogen levels produces greater neuronal and local vascular damage, resulting in a higher chance of postthrombolysis sICH. Bagoly et al's review article demonstrated that 
the assessment of pre- and postthrombolysis plasma fibrinogen levels was potentially useful to predict postthrombolysis sICH but more data are needed to establish its association with unfavorable outcome. ${ }^{24}$

The study was limited by small sample size, suffers from the probability of selection bias for not including a control group consisting of patients not receiving thrombolytic treatment, our inability to estimate FDP levels in all patients, lack of a long-term follow-up, and lack of MRIbased protocols prior to thrombolysis, thereby, hindering our ability to test leukoaraiosis, microbleeds, and low ADC coefficient as predictors of sICH. Therefore, the results should be confirmed in multicenter cohort studies.

Hence, this study made the following conclusions:

1. Independent predictors of unfavorable outcome after thrombolysis were past TIA, CHADS2 score $>2$, occurrence of TACS, low EF, higher age, MABP, CBG, and NIHSS score.

2. Independent predictors of sICH were CHADS2 score $>2$, occurrence of TACS, AF, low EF, ongoing antiplatelet and anticoagulant therapy, higher MABP, CBG, SEDAN, and NIHSS scores and lower ASPECTS score.

Thus, CHADS2 score $>2$, low EF, occurrence of TACS and higher NIHSS score, MABP, and CBG at admission were the common risk factors significantly predicting postthrombolysis sICH and morbidity.

3. Higher fibrinogen levels were significantly related with higher rates of sICH, morbidity, and mortality. After thrombolysis, the level of fibrinogen significantly reduced. Thus, it is a potential biomarker.

4. The performance of thrombolysis within 3 hours or between 3 and 4.5 hours after the onset of symptoms did not affect morbidity, mortality, or the occurrence of sICH.

\section{Ethical Approval}

Institutional Ethics clearance granted for the study.

\section{Authors' Contributions}

G.P.M. and S.D. developed the concept. R.B. designed the study. S.D., H.K.P., S.A.P., and C.P. collected the data. K.C.G. conducted literature overview and involved in discussion. S.D. performed statistical analysis.

\section{Funding}

None.

\section{Conflict of Interest}

None declared.

\section{References}

1 Ghandehari K. Barriers of thrombolysis therapy in developing countries. Stroke Res Treat 2011;2011:686797

2 Kamalakannan S, Gudlavalleti ASV, Gudlavalleti VSM, Goenka $\mathrm{S}$, Kuper H. Incidence \& prevalence of stroke in India: a systematic review. Indian J Med Res 2017;146(2):175-185

3 Sudlow CLM, Warlow CP; International Stroke Incidence Collaboration. Comparable studies of the incidence of stroke and its pathological types: results from an international collaboration. Stroke 1997;28(3):491-499
4 Lee HJ, Lee JS, Choi JC, et al. Simple estimates of symptomatic intracranial hemorrhage risk and outcome after intravenous thrombolysis using age and stroke severity. J Stroke 2017;19(2):229-231

5 Liu M, Pan Y, Zhou L, Wang Y. Predictors of post-thrombolysis symptomatic intracranial hemorrhage in Chinese patients with acute ischemic stroke. PLoS One 2017;12(9):e0184646

6 Chenna V, Kaul S, Tandra S, et al. Predictors of intracerebral hemorrhage in acute stroke patients receiving intravenous recombinant tissue plasminogen activator. Ann Indian Acad Neurol 2018;21(3):214-219

7 Jickling GC, Sharp FR. Blood biomarkers of ischemic stroke. Neurotherapeutics 2011;8(3):349-360

8 Bamford J, Sandercock P, Dennis M, Burn J, Warlow C. Classification and natural history of clinically identifiable subtypes of cerebral infarction. Lancet 1991;337(8756): 1521-1526

9 Muengtaweepongsa S, Prapa-Anantachai P, Dharmasaroja PA, Rukkul P, Yodvisitsak P. External validation of the SEDAN score: the real world practice of a single center. Ann Indian Acad Neurol 2015;18(2):181-186

10 European Stroke Organisation (ESO) Executive Committee ESO Writing Committee. Guidelines for management of ischaemic stroke and transient ischaemic attack 2008. Cerebrovasc Dis 2008;25(5):457-507

11 Hsieh CY, Chen CH, Chen YC, Kao Yang YH. National survey of thrombolytic therapy for acute ischemic stroke in Taiwan 2003-2010. J Stroke Cerebrovasc Dis 2013;22(8):e620-e627

12 Çetiner M, Aydin HE, Güler M, Canbaz Kabay S, Zorlu Y. Predictive factors for functional outcomes after intravenous thrombolytic therapy in acute ischemic stroke. Clin Appl Thromb Hemost 2018;24(9 suppl) :171S:177S

13 Zhao Q Li X, Dong W, et al. Factors associated with thrombolysis outcome in ischemic stroke patients with atrial fibrillation. Neurosci Bull 2016;32(2):145-152

14 Huang YH, Zhuo ST, Chen YF, et al. Factors influencing clinical outcomes of acute ischemic stroke treated with intravenous recombinant tissue plasminogen activator. Chin Med J (Engl) 2013;126(24):4685-4690

15 Bateman BT, Schumacher HC, Boden-Albala B, et al. Factors associated with in-hospital mortality after administration of thrombolysis in acute ischemic stroke patients: an analysis of the nationwide inpatient sample 1999 to 2002. Stroke 2006;37(2):440-446

16 Heuschmann PU, Kolominsky-Rabas PL, Roether J, et al; German Stroke Registers Study Group. Predictors of in-hospital mortality in patients with acute ischemic stroke treated with thrombolytic therapy. JAMA 2004;292(15):1831-1838

17 Padma MV, Singh MB, Bhatia R, et al. Hyperacute thrombolysis with IV rtPA of acute ischemic stroke: efficacy and safety profile of 54 patients at a tertiary referral center in a developing country. Neurol India 2007;55(1):46-49

18 Sharma SR, Sharma N. Hyperacute thrombolysis with recombinant tissue plasminogen activator of acute ischemic stroke: feasibility and effectivity from an Indian perspective. Ann Indian Acad Neurol 2008;11(4):221-224

19 Salam KA, Ummer K, Kumar VG, Noone ML, Laila A, Ragini $\mathrm{J}$. Intravenous thrombolysis for acute ischemic stroke: the Malabar experience 2003 to 2008. J Clin Neurosci 2009;16(10):1276-1278

20 Boddu DB, Srinivasarao Bandaru VC, Reddy PG, et al. Predictors of major neurological improvement after intravenous thrombolysis in acute ischemic stroke: a hospital-based study from south India. Neurol India 2010;58(3):403-406

21 Whiteley WN, Emberson J, Lees KR, et al; Stroke Thrombolysis Trialists' Collaboration. Risk of intracerebral haemorrhage with alteplase after acute ischaemic stroke: a secondary 
analysis of an individual patient data meta-analysis. Lancet Neurol 2016;15(9):925-933

22 Albers GW, Clark WM, Madden KP, Hamilton SA. ATLANTIS trial: results for patients treated within 3 hours of stroke onset. Alteplase thrombolysis for acute noninterventional therapy in ischemic stroke. Stroke 2002;33(2):493-495

23 Modrego PJ. The risk of symptomatic intracranial hemorrhage after thrombolysis for acute stroke: current concepts and perspectives. Ann Indian Acad Neurol 2019;22(3):336-340

24 Bagoly Z, Szegedi I, Kálmándi R, Tóth NK, Csiba L. Markers of coagulation and fibrinolysis predicting the outcome of acute ischemic stroke thrombolysis treatment: a review of the literature. Front Neurol 2019;10:513

25 Huang X, Moreton FC, Kalladka D, et al. Coagulation and fibrinolytic activity of tenecteplase and alteplase in acute ischemic stroke. Stroke 2015;46(12):3543-3546
26 Martí-Fàbregas J, Borrell M, Cocho D, et al. Hemostatic markers of recanalization in patients with ischemic stroke treated with rt-PA. Neurology 2005;65(3):366-370

27 Ill-Raga G, Palomer E, Ramos-Fernández E, et al. Fibrinogen nitrotyrosination after ischemic stroke impairs thrombolysis and promotes neuronal death. Biochim Biophys Acta 2015;1852(3):421-428

28 Han HS, Qiao Y, Karabiyikoglu M, Giffard RG, Yenari MA. Influence of mild hypothermia on inducible nitric oxide synthase expression and reactive nitrogen production in experimental stroke and inflammation. J Neurosci 2002;22(10):3921-3928

29 Iadecola C, Zhang F, Casey R, Nagayama M, Ross ME. Delayed reduction of ischemic brain injury and neurological deficits in mice lacking the inducible nitric oxide synthase gene. J Neurosci 1997;17(23):9157-9164 